\title{
Liquid Sloshing in a Rotating, Laterally Oscillating Cylindrical Container
}

\author{
Yusuke Saito, Tatsuo Sawada* \\ Department of Mechanical Engineering, Keio University, Japan
}

Copyright $\bigcirc 2017$ by authors, all rights reserved. Authors agree that this article remains permanently open access under the terms of the Creative Commons Attribution License 4.0 International License

\begin{abstract}
Free surface movement of water in a rotating, laterally oscillating cylindrical container was qualitatively investigated. Time-dependent dynamic pressure was measured instead of free surface displacement. The swirling direction was determined by forcing the frequency and rotating direction of the cylindrical container. The swirling direction was opposite to that of the rotating cylindrical container when the forcing frequency was low, whereas the crest of the free surface swirled in the same direction as that of the rotating cylindrical container when the forcing frequency greatly increased. Unstable swirling occurred when swirling direction changes, but it disappeared as the rotating frequency increased.
\end{abstract}

Keywords Sloshing, Rotating Cylindrical Container, Swirling, Pressure Measurement

\section{Background}

Sloshing is when liquids with a free surface oscillate in a container. It is caused by disturbances in a partially filled liquid container. Sloshing is a major problem in engineering, e.g., petroleum tank in an earthquake and in an LNG tanker. In particular, sloshing in axisymmetric containers occurs when surface waves rotate around the center axis of a container [1]. This phenomenon is called "swirling." This is also a cause of concern for containers with large capacities, such as liquid fuel tanks in rockets and missiles, since they contain a large amount of liquid propellant. Various theoretical, experimental, and numerical researches for sloshing have been conducted and have been summarized by Ibrahim [2].

Fluid dynamic behavior in spinning tanks is related to the stability and control of rockets and spacecraft. Yam et al. [3] considered the stability of a spinning axisymmetric rocket having dissipative internal fluid motion. Bauer and Eidel [4] and Zhang et al. [5] examined free surface oscillations in a slowly spinning cylindrical container partially filled with a viscous fluid. Ohaba et al. [6] investigated the frequency response of liquid surface in a rotating, laterally oscillating cylindrical container and demonstrated that cylindrical container rotation stabilizes the swirling. However, further detailed experiments have not been conducted thereafter.

In this study, we measure the time-dependent dynamic pressure of water in a rotating, laterally oscillating cylindrical container instead of the free surface displacement. There are several experimental parameters in the experimental system, e.g., forcing frequency, rotating frequency of the cylindrical container, and water depth. Here, we present the qualitative influence of rotating frequency of the cylindrical container on sloshing and swirling of water to understand free surface behavior.

\section{Experimental Configuration}

Figure 1 shows the experimental apparatus, and a cylindrical container is shown in Fig. 2. The cylindrical container is made of Plexiglas; its height and inner diameter are 200 and $99 \mathrm{~mm}$, respectively. The cylindrical container is connected to a motor fixed on the oscillating table. This table is sinusoidally oscillated in the horizontal direction by the vibration generator. Therefore, the cylindrical container laterally oscillates while it rotates. The lateral forcing frequency $f$ is continuously changed from 1.0 to $5.0 \mathrm{~Hz}$ by the wave generator. The amplitude of the lateral oscillation is $X_{0}=1.0 \mathrm{~mm}$, and the water depth is $h=50 \mathrm{~mm}$ for all experiments. The rotating frequency of the cylindrical container and displacement of the oscillating table is measured by a photoelectric sensor and laser displacement sensor, respectively. 


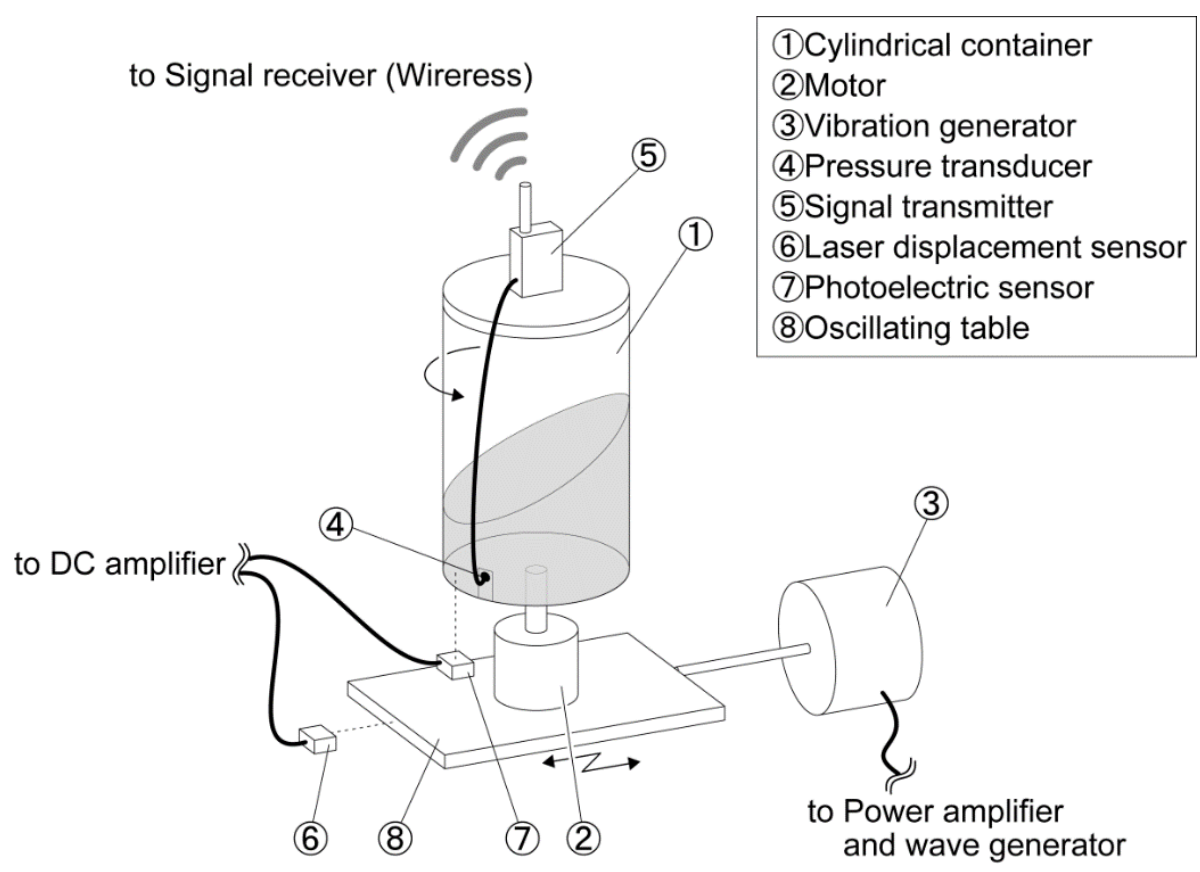

Figure 1. Experiment apparatus

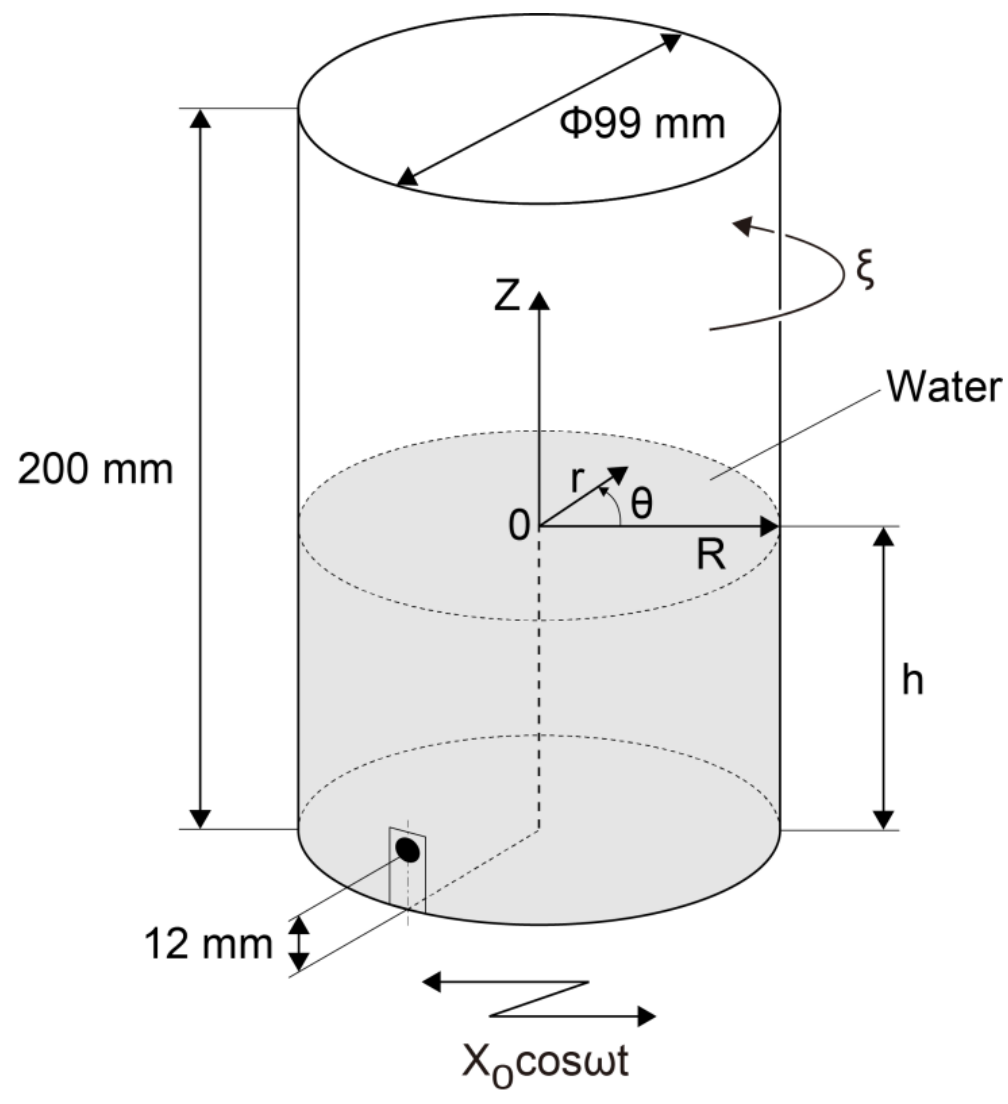

Figure 2. Cylindrical container 
We verified that free surface displacement corresponds to fluid dynamic pressure from our previous measurements [7]. The pressure sensor is embedded in the inner wall of the cylindrical container, as shown in Fig. 2. Its vertical position is $12 \mathrm{~mm}$ from the bottom wall. As the cylindrical container is rotating, a wireless signal processing system is introduced.

During sloshing, the dynamic pressure measured by the pressure sensor fluctuated. In one period of the lateral oscillation of the cylindrical container, the difference between the maximum and the minimum pressure is measured as $\Delta p . \Delta p$ is measured 15 times for each forcing frequency $f$.

\section{Theoretical Approach}

Our analytical model is shown in Fig. 2, where $z$ is vertically measured upward from the mean position of the free surface; $\theta=0$ indicates the direction of the forced oscillation. $R$ is the radius of the cylindrical container, $\xi$ is the angular velocity of the cylindrical container, and $\omega$ is the angular frequency of the oscillating table. Assuming irrotational flow and an incompressible fluid, and considering a coordinate system fixed to the cylindrical container, the unsteady irrotational Bernoulli equation for $z=\eta(r, \theta, t)$ is given by

$$
\begin{gathered}
\frac{\partial \phi}{\partial t}+\frac{1}{2}|\nabla \phi|^{2}+\frac{p}{\rho}+\Pi=0 \\
\Pi=-\frac{X_{0} r}{2}(\omega-\xi)^{2} \cos [(\omega-\xi) t+\theta]-\frac{X_{0} r}{2}(\omega+ \\
\xi)^{2} \cos [(\omega+\xi) t-\theta]+g z
\end{gathered}
$$

where $\phi, p, \rho, \Pi$ and $g$ are velocity potential, water pressure, water density, external force potential, and gravitational acceleration, respectively. For infinitesimally small waves, $|\nabla \phi|^{2}$ is assumed to be negligible; Using Eq. (2), the kinematic and dynamic free surface conditions are given by the following equations:

$$
\begin{gathered}
\frac{\partial \eta}{\partial t}=\left(\frac{\partial \phi}{\partial z}\right)_{z=0} \\
\left(\frac{\partial^{2} \phi}{\partial t^{2}}+g \frac{\partial \phi}{\partial z}\right)_{\substack{\left.z=0 \\
(\omega+\xi)^{3} \cos [(\omega+\xi) t-\theta]\right\}}}=\frac{X_{0} r i}{2}\left\{(\omega-\xi)^{3} \cos [(\omega-\xi) t+\theta]+\right.
\end{gathered}
$$

where $\eta$ is liquid surface displacement. Boundary conditions on the bottom and side walls are given by

$$
\begin{gathered}
\left(\frac{\partial \phi}{\partial z}\right)_{z=-h}=0 \\
\left(\frac{\partial \phi}{\partial r}\right)_{r=R}=0
\end{gathered}
$$

The continuity equation $\nabla^{2} \phi=0$ is solved by using boundary conditions (3) - (6) and the velocity potential is indicated by

$$
\begin{gathered}
\phi= \\
\sum_{m=1}^{\infty} \sum_{n=1}^{\infty}\left\{A _ { m } \operatorname { c o s h } \left[k_{m n}(z+\right.\right. \\
h)] J_{m}\left(k_{m} r\right) \cos \left(m \theta+\delta_{m n}\right) \cos \left(\omega_{m n} t+\epsilon_{m n}\right)- \\
\frac{\omega_{m n}^{2}(\omega+\xi)^{3}}{\omega_{m n}^{2}-(\omega+\xi)^{2}} \frac{r X_{0} \cosh \left[k_{m n}(z+h)\right]}{2 g k_{m n} \sinh \left(k_{m n} h\right)} \sin [(\omega-\xi) t+\theta]- \\
\left.\frac{\omega_{m n}^{2}(\omega-\xi)^{3}}{\omega_{m n}^{2}-(\omega-\xi)^{2}} \frac{r X_{0} \cosh \left[k_{m n}(z+h)\right]}{2 g k_{m n} \sinh \left(k_{m n} h\right)} \sin [(\omega+\xi) t-\theta]\right\}
\end{gathered}
$$

where $m$ and $n$ are natural numbers $(m=1,2,3, \cdots ; n=$ $1,2,3, \cdots)$ that represent vibrational modes. $J_{m}$ is the Bessel function of the first kind of order $m . A_{m n}, \delta_{m n}$, and $\epsilon_{m n}$ are arbitrary constants. Here, $k_{m n}$ is a constant that satisfies the following equation:

$$
\left[\frac{d}{d r} J_{m}\left(k_{m n} r\right)\right]_{r=R}=0
$$

and $\omega_{m n}$ is the characteristic angular frequency given by

$$
\omega_{m n}=\sqrt{g k_{m n} \tanh \left(k_{m n} h\right)}
$$

In Eq. (7), the second and third terms are significant in comparison with the first term in the vicinity of the resonant frequency. Therefore, the first term in Eq. (7) can be ignored. The higher-order vibrational mode is also ignored because it hardly appears. We adopt only the $(1,1)$ mode. Considering the coordinate system fixed to the ground, the liquid surface displacement $\eta$ is calculated by Eq. (1):

$$
\eta=\frac{\omega_{11}^{2}(\omega+\xi)^{2}}{\omega_{11}^{2}-(\omega+\xi)^{2}} \frac{r X_{0}}{2 g} \cos (\theta-\omega t)+\frac{\omega_{11}^{2}(\omega-\xi)^{2}}{\omega_{11}^{2}-(\omega-\xi)^{2}} \frac{r X_{0}}{2 g} \cos (\theta+
$$

Equation (10) is considered in the coordinate system fixed to the ground. Figure 3 shows the calculated frequency response of the liquid surface displacement $\eta$ and each term in Eq. (10). Here, $\psi=\xi / 2 \pi$ is the rotating frequency of the cylindrical container. The swirling direction is indicated in Fig. 3.

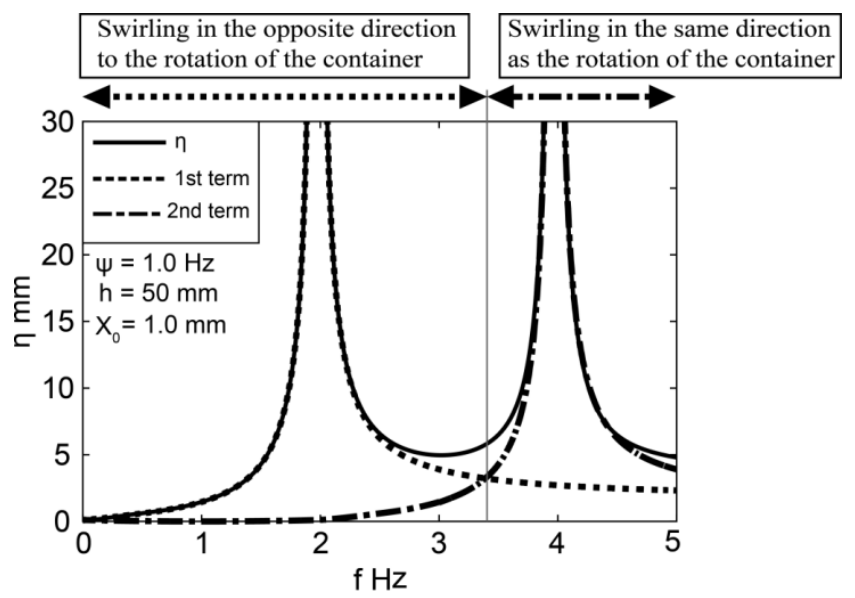

Figure 3. Frequency response of liquid surface fluctuation 


\section{Results and Discussion}

We define the following non-dimensionalized pressure $p^{*}$ to arrange measured pressure change as

$$
p^{*}=\frac{\Delta p}{\rho g R}
$$

We present the swirling phenomena of the cylindrical container before we indicate experimental results (see Fig. 4). When the forcing frequency is less, the free surface vibrates in the same direction as that of the forced oscillation. The surface elevation gradually increases with increase in the forcing frequency, and the free surface forms a collapsed wave near the boundary when the swirling direction changes. After this point, the crest of the free surface rotates around the center axis of the cylindrical container. The direction of the rotation is not fixed, and the clockwise $(\mathrm{CW})$ and counterclockwise $(\mathrm{CCW})$ swirling are irregularly repeated. This is called "unstable swirling." The surface elevation decreases with the forcing frequency, and the unstable swirling changes to swirling wherein the direction is fixed. This is called "stable swirling." The rotational direction is constant, but it is dependent on some disturbance in the experimental system, e.g., roundness of the cylindrical container and small oscillating components of the oscillating table. When the forcing frequency becomes much larger, the rotation of the free surface disappears, and the swirling changes to an oscillation similar to that of the initial lateral vibration.

Figure 5 shows the frequency response of pressure change without rotation of the cylindrical container. Here, $\gamma$ is the circumferential position of the sensor, and $\gamma$ corresponds to the $\theta$-coordinate in Fig. 2 . Since the free surface motion is a sinusoidal oscillation before the unstable swirling, pressure change for $\gamma=0$ gradually increases with the forcing frequency; while pressure change for $\gamma=\pi / 2$ does not increase until the unstable swirling begins. Pressure changes for both $\gamma=0$ and $\pi / 2$ indicate irregular fluctuation when the swirling direction changes. When the forcing frequency increases, stable swirling begins and the pressure changes increase in this region. When the stable swirling disappears, pressure changes suddenly decrease.

Figures 6 and 7 present frequency responses of pressure change with rotation of the cylindrical container. The rotational direction of the stable swirling is the same as that of the cylindrical container. We provide experimental results for $\mathrm{CW}$ rotation after this because the transition of the pressure changes hardly depends on the rotational direction of the cylindrical container, as shown in Figs. 6 and 7.

Frequency responses of pressure change for $\psi=$ $0.5 \mathrm{~Hz}, 1.0 \mathrm{~Hz}$, and $1.5 \mathrm{~Hz}$ are shown in Figs. 8, 9, and 10, respectively. Observing the start/stop oscillating frequency of the stable swirling is sometimes difficult. We use dotted lines in Figs. 8, 9, and 10 in such cases. When the rotation of the cylindrical container exists, the pressure change greatly decreases in comparison with that in Fig. 5. This indicates that the disturbance of the free surface is suppressed by the rotation of the cylindrical container. As $\psi$ increases, a no-swirling region occurs near $f=3.0 \mathrm{~Hz}$. In this region, the free surface is laterally oscillating, but its direction is vertical to that of the forced oscillation. Though the change of the oscillating direction is very interesting from a research viewpoint, we refer to it at another time. The unstable swirling disappears after $\psi=1.0 \mathrm{~Hz}$.

For values of $\psi$ greater than $1.0 \mathrm{~Hz}$, the stable swirling first appears when the forcing frequency increases. If the rotational direction of the cylindrical container is clockwise, the direction is always counterclockwise. The reverse is also true.

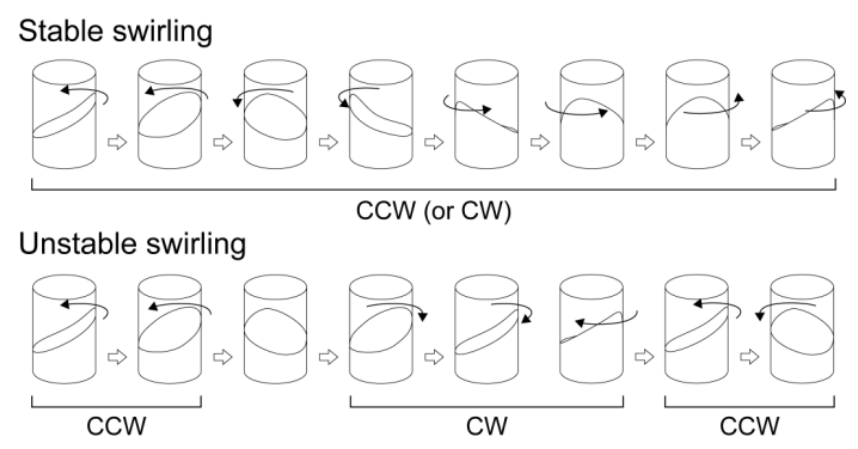

Figure 4. Stable and unstable swirling

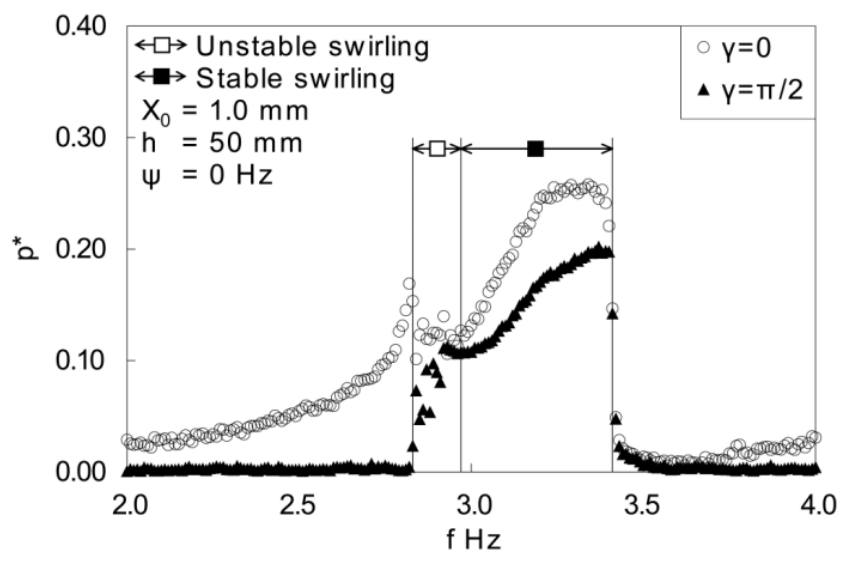

Figure 5. Experimental results for frequency response of pressure fluctuation for $\psi=0.0 \mathrm{~Hz}$

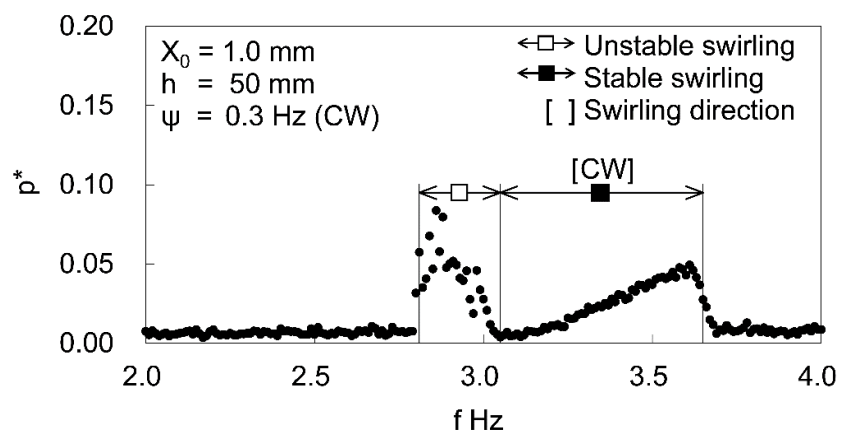

Figure 6. Experimental results for frequency response of pressure fluctuation for $\psi=0.3 \mathrm{~Hz}(\mathrm{CW})$ 


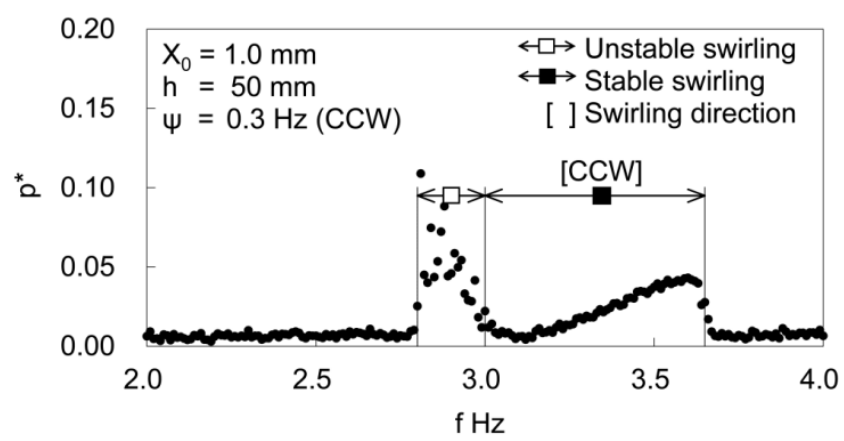

Figure 7. Experimental results for frequency response of pressure fluctuation for $\psi=0.3 \mathrm{~Hz}(\mathrm{CCW})$

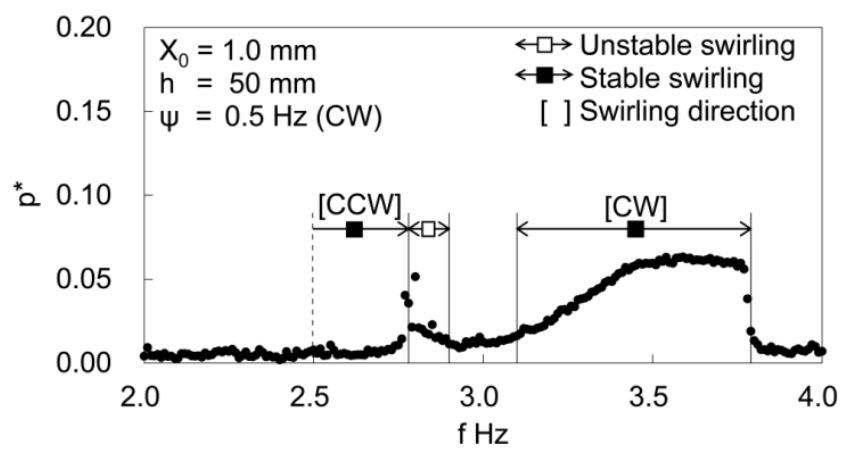

Figure 8. Experimental results for frequency response of pressure fluctuation for $\psi=0.5 \mathrm{~Hz}(\mathrm{CW})$

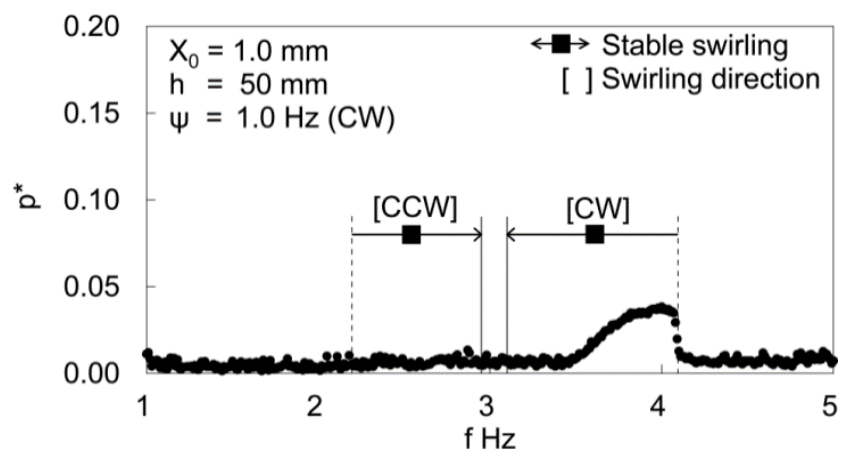

Figure 9. Experimental results for frequency response of pressure fluctuation for $\psi=1.0 \mathrm{~Hz}(\mathrm{CW})$

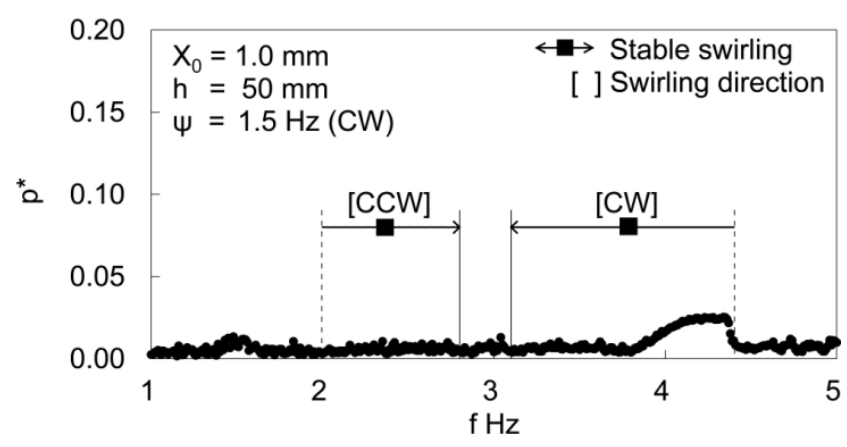

Figure 10. Experimental results for frequency response of pressure fluctuation for $\psi=1.5 \mathrm{~Hz}(\mathrm{CW})$

\section{Concluding Remarks}

We examined the frequency response of the dynamic pressure change of water in an upright, rotating, laterally oscillating cylindrical container to understand the relation between the sloshing and swirling of water. The sloshing motion of the water was suppressed by adding a rotational motion to the lateral oscillation of the cylindrical container. Moreover, unstable swirling disappeared as the rotating frequency increased. When the forcing frequency increased, the crest of free surface swirled and its direction varied dependent on the rotational direction of the cylindrical container. The swirling direction was uniquely determined by the rotating and forcing frequencies. As mentioned above, we have obtained several unique interesting experimental results from the measurement of the time-dependent dynamic pressure. The frequency response and the direction of swirling derived by linear theoretical analysis are roughly in agreement with experimental results. The swirling contains two kinds of wave component from the theoretical analysis. However, it was difficult to clarify existence of these wave components in the present experiment.

\section{Acknowledgements}

We would like to thank to Mr. T. Seki of Tokushu Keisoku, Co. Ltd. for his assistance in the experiments. We also wish to acknowledge the contribution of Mr. H. Kinoshita who was a student at Keio University.

\section{REFERENCES}

[1] R. E. Hutton, An investigation of resonant, nonlinear, non-planar free surface oscillations of a fluid, NASA Tech Note D-1870, (1963).

[2] R. A. Ibrahim, Liquid sloshing dynamics, Part I, (2005), Cambridge University Press.

[3] Y. Yam, D. L. Mingori, and D. M. Halsmer, Stability of a spinning axisymmetric rocket with dissipative internal mass motion, Journal of Guidance, Control, and Dynamics, Vol. 20 (1997), pp. 306-312.

[4] H. F. Bauer and W. Eidel, Axisymmetric damped natural frequencies in a slowly spinning cylindrical container filled with viscous liquid, Forschung im Ingenieurwesen, Vol. 67 (2002), pp. 93-99.

[5] E. Zhang, J. Tang, and M. Tao, Dynamic stability of a rotor filled or partially filled with liquid, Journal of Applied Mechanics, Vol. 63 (1996), pp. 101-105.

[6] M. Ohaba et al., Liquid sloshing response in a spinning cylindrical container, Proceedings of JSME Fluids Engineering Conference, (1995), pp. 203-204, (in Japanese).

[7] T. Ishiyama et al., Relation between the dynamic pressure and displacement of the free surface in two-layer sloshing with magnetic fluids and silicone oil, Materials Science Forum, Vol. 792 (2014), pp. 33-38. 